\title{
Activation of Early Silent Synapses by Spontaneous Synchronous Network Activity Limits the Range of Neocortical Connections
}

\author{
Thomas Voigt, Thoralf Opitz, and Ana Dolabela de Lima \\ Department of Developmental Physiology, Otto-von-Guericke University, Institute of Physiology, 39120 Magdeburg, Germany
}

\begin{abstract}
During the early development of neocortical networks, many glutamatergic synapses lack AMPA receptors and are physiologically silent. We show in neocortical cultures that spontaneous synchronous network activity is able to convert silent synapses to active synapses by the incorporation of AMPA receptors into synaptic complexes throughout the network within a few minutes. To test the effect of synaptic activation on the connectivity of neuronal populations, we created separated neuronal networks that could innervate each other. We allowed outgrowing axons to invade the neighboring network either before or after the onset of synchronous network activity. In the first case, both subnetworks connected to each other and synchronized their activity, whereas in the second case, axonal connections failed to form and network activity did not synchronize between compartments. We conclude that early spontaneous synchronous network activity triggers a global AMPAfication of immature synapses, which in turn prevents later-arriving axons from forming afferent connections. This activity-dependent process may set the range of corticocortical connections during early network development before experience-dependent mechanisms begin elaborating the mature layout of the neocortical connections and modules.
\end{abstract}

Key words: cerebral cortex; cell culture; development; glutamate receptors; silent synapses; synchronous activity

\section{Introduction}

In all brain structures investigated so far, large-scale synchronous network activity has been observed for a limited period of development (Ben-Ari et al., 1997; Garaschuk et al., 1998; O’Donovan, 1999). Spontaneous synchronous network activity represents long bursts of firing occurring concurrently across the network with a frequency of $<0.5 \mathrm{~Hz}$. This activity is often defined experimentally based on calcium imaging experiments as a lowfrequency rise of the intracellular calcium concentration $\left(\left[\mathrm{Ca}^{2+}\right]_{\mathrm{i}}\right)$ in many neurons simultaneously (Garaschuk et al., 2000; Ben-Ari, 2001; Voigt et al., 2001). In correlated imaging and patch-clamp experiments, it has been shown that calcium transients are generated by barrages of activity in the network. When $\left[\mathrm{Ca}^{2+}\right]_{\mathrm{i}}$ increased synchronously in a large number of neurons in the field under observation, the electrophysiological recording of single cells displayed a burst of action potentials riding on top of a long-lasting depolarization (Murphy et al., 1992; Wong, 1998; Opitz et al., 2002).

Slow synchronous network activity develops in immature neocortical networks shortly after neurogenesis and disappears before the onset of experience-dependent plasticity. Interest-

Received Sept. 14, 2004; revised March 23, 2005; accepted March 26, 2005

This work was supported by Deutsche Forschungsgemeinschaft Grant V0519/6-1 and Land Sachsen Anhalt Grant 3431A/0302M. We thank B. Adam and A. Reupsch for their expert technical assistance and B. D. Lima for helpful comments on a previous version of this manuscript. We also thank the anonymous reviewers for their constructive criticism.

Correspondence should be sent to Dr. Thomas Voigt, Otto-von-Guericke University, Institute of Physiology, Leipziger Straße 44, D-39120 Magdeburg, Germany. E-mail: thomas.voigt@medizin.uni-magdeburg.de.

DOI:10.1523/JNEUROSCI.3803-04.2005

Copyright $\odot 2005$ Society for Neuroscience $\quad$ 0270-6474/05/254605-11\$15.00/0 ingly, in a similar timetable [postnatal day 0 (P0) to P8], the existence of large amounts of silent synapses has been described in many brain structures (Malenka and Nicoll, 1997). Those young synapses contain only NMDA receptors (NMDARs), making them functionally silent at resting membrane potential (Durand et al., 1996; Wu et al., 1996; Isaac et al., 1997; Bardoni et al., 1998; Hsia et al., 1998; Li and Zhuo, 1998; Rumpel et al., 1998; Petralia et al., 1999; Lu et al., 2001). Silent synapses containing only NMDA receptors are transformed within minutes into activated synapses containing both NMDA and AMPA receptors (AMPAfication) by simultaneous presynaptic and postsynaptic depolarization (Durand et al., 1996; Wu et al., 1996; Isaac et al., 1997; Shi et al., 1999; Pickard et al., 2000; Liao et al., 2001).

It has been hypothesized that the synchronous long-range calcium waves early in development have a function in the establishment of long-range connections (Garaschuk et al., 2000) and are related to the synchronization of synaptogenesis throughout the cortex (Voigt et al., 1997, 2001). By the time large-scale oscillatory calcium waves have ceased in the cerebral cortex, the number of silent synapses has dropped markedly (Isaac et al., 1997; Garaschuk et al., 2000). Thus, it is possible that this generalized activation of immature synapses may provide a basis for synchronizing later phases of synaptogenesis, not only influencing the formation of corticocortical connections (Schwindt et al., 1988; Durand et al., 1996; Isaac et al., 1997; Rumpel et al., 1998; Feller, 1999; O’Donovan, 1999; Ben-Ari, 2001; Losi et al., 2002) but also contributing to the transition between spontaneous activitydependent and sensorial experience-dependent development.

Here we tested the hypothesis that the two mechanisms, acti- 
vation of silent synapses and the occurrence of large-scale synchronized activity, are functionally correlated and influence the generation of long-range interconnections in young cortical neuronal networks that develop from dissociated embryonic day 16 (E16) rat cortices.

\section{Materials and Methods}

Cell culture. Cortical neurons were cultivated in serum free medium (N2) in the presence of a surrounding glial feeding layer. All experimental procedures were approved by local government authorities.

Each acid cleaned coverslip was fitted to a $20 \mathrm{~mm}$ hole in the bottom of a $60 \mathrm{~mm}$ Petri dish and was treated overnight with poly-D-lysine $(0.1$ $\mathrm{mg} / \mathrm{ml}$ in borate buffer, $\left.\mathrm{pH} 8.5,36^{\circ} \mathrm{C}\right)$. Astroglial cultures were prepared from cerebral hemispheres of P0-P3 Sprague Dawley rats as reported in detail previously (de Lima and Voigt, 1999). Purified astroglial cells were plated at a density of 300 cells $/ \mathrm{mm}^{2}$ in the outer portion of the Petri dish bottom $5 \mathrm{~d}$ before the neurons. To prevent the glial cells from spreading onto the coverslip, the central region was temporarily isolated by a stainless steel ring glued with silicon grease around the hole.

Neuronal cultures were prepared from cerebral cortices of embryonic Sprague Dawley rats at E16 (day after insemination was E1). The cells were taken from the dorsolateral parts of the telencephalic vesicles (excluding hippocampal and basal telencephalic anlagen), dissociated with trypsin/EDTA, and seeded onto the poly-D-lysine-coated glass coverslips at a density of $200-250$ cells $/ \mathrm{mm}^{2}$ in N2 medium (Bottenstein and Sato, 1979) (75\% DMEM, 25\% Ham's F12, and N2 supplements; Invitrogen, Carlsbad, CA). Cultures were treated at $4 \mathrm{~d}$ in vitro (DIV) with cytosine arabinoside at a final concentration of $5 \mu \mathrm{M}$, and a third of the medium volume was then changed after $24 \mathrm{~h}$. All cultures were maintained in a humidified $5 \% \mathrm{CO}_{2} / 95 \%$ air atmosphere at $36^{\circ} \mathrm{C}$.

Drugs and drug treatment. To raise cultures with blocked GABAergic transmission, $20 \mu \mathrm{M}$ (-)bicuculline methiodide (BMI) (Sigma/RBI, Deisenhofen, Germany) was added to the culture medium at 5 DIV, at 8 or 9 DIV, and at 11 or 13 DIV. For immunocytochemistry, cultures were fixed in the presence of BMI. Imaging experiments (Fluo-3 loading and recording) were also performed in the presence of the blocker, unless otherwise stated. For the blockade of glutamatergic synaptic transmission, either 6-cyano-7-nitroquinoxaline-2,3-dione disodium (CNQX) (10 $\mu \mathrm{M}$; Tocris Cookson, Ellisville, MO) or D-2-amino-5phosphonopentanoic acid (APV) (50 $\mu \mathrm{M}$; Tocris Cookson) was used.

For $0 \mathrm{~mm} \mathrm{Mg}^{2+}$ stimulation, culture medium was replaced by $0 \mathrm{~mm}$ $\mathrm{Mg}^{2+}$ HEPES-buffered Ringer's solution (in mM: $140 \mathrm{NaCl}, 5 \mathrm{KCl}, 0.75$ $\mathrm{CaCl}_{2}, 1.25 \mathrm{NaH}_{2} \mathrm{PO}_{4}, 20$ D-glucose, 30 glycine, and $15 \mathrm{HEPES} / \mathrm{NaOH}$, $\mathrm{pH}$ 7.4) either $3 \mathrm{~h}$ before fixation (receptor immunocytochemistry experiments) or immediately before recording (imaging experiments), unless otherwise described in Results.

For the complete block of glutamate- and GABA-mediated synaptic transmission, a mixture of glutamate receptor antagonists CNQX (10 $\mu \mathrm{M}$; Tocris Cookson) and APV (50 $\mu \mathrm{M}$; Tocris Cookson) and $\mathrm{GABA}_{\mathrm{A}}$ receptor $\left(\mathrm{GABA}_{\mathrm{A}} \mathrm{R}\right)$ antagonists $\mathrm{BMI}(20 \mu \mathrm{M}$; Sigma/RBI) and picrotoxin (PTX) (10 $\mu \mathrm{M}$; Tocris Cookson) were added to the cultures at 5 DIV and refreshed at 9 and 11 DIV. This drug mixture was removed from the cultures at 13 DIV by a brief wash with DMEM, followed by the addition of drug-free glia-conditioned N2 medium. The cultures survived in the drug-free medium for another 1-3 DIV before paired patch-clamp recordings.

Neurotransmitter receptor immunocytochemistry. For immunocytochemical receptor staining, cell cultures were fixed for $30 \mathrm{~min}$ at $36^{\circ} \mathrm{C}$ in $4 \%$ paraformaldehyde in phosphate buffer $(0.1 \mathrm{M}, \mathrm{pH} 7.4)$ containing $0.12 \mathrm{M}$ glucose, followed by chilled methanol $\left(-20^{\circ} \mathrm{C}\right)$ for $10 \mathrm{~min}$ at $4^{\circ} \mathrm{C}$. After washing in PBS (0.01 M, pH 7.4), cultures were incubated in $0.25 \%$ Triton X-100, 10\% goat serum, 5\% sucrose, and 2\% BSA in PBS for 10 min at room temperature. For double staining of NMDA receptor subunit NR1 and glutamate receptor subunits GluR2/3, cell cultures were incubated with a mixture of monoclonal mouse anti-NR1 (1:250; PharMingen, Heidelberg, Germany) and polyclonal rabbit anti-GluR2/3 (1:100; PharMingen or Chemicon, Hofheim, Germany) overnight at $4^{\circ} \mathrm{C}$. In most experiments, a polyclonal rabbit anti-GluR1 (1:50; Chemi- con) was used either in a NR1/GluR1 combination or in a NR1/GluR2/ $3 /$ GluR1 combination. The pooled primary antibodies were revealed in few experiments with a mixture of goat anti-rabbit indocarbocyanine 3 (Cy3) and goat anti-mouse carbocyanine (Cy2) (1:400; Jackson ImmunoResearch, West Grove, PA) for $2 \mathrm{~h}$ at room temperature. In most experiments, however, the double staining was performed sequentially by first staining anti-NR1, followed by goat anti-mouse Cy2, and then, after a short fixation $\left(30 \mathrm{~min}, 4 \%\right.$ paraformaldehyde, $36^{\circ} \mathrm{C}$ ), antiGluR2/3 was followed by goat anti-rabbit Cy3. The sequential application of antibodies yielded in our hands more consistent results and the strongest signals of both antibodies. Cell cultures were rinsed thoroughly with PBS between every incubation step. To detect synaptic localization of the receptor staining, the anti-NR1 antibody was combined with a polyclonal rabbit anti-synaptophysin antibody (1:500; Synaptic Systems, Göttingen, Germany). Goat anti-mouse Cy2 and goat anti-rabbit Cy3 were used as secondary antibody. After a final wash in PBS, coverslips were dehydrated in an ethanol series, cleared in two changes of xylene, and mounted on clean slides with Fluoromount (BDH Laboratory Supplies, Poole, UK).

Analysis of receptor staining. For quantitative evaluation of the receptor staining, at least two coverslips were analyzed per time point and experimental condition in each of at least two experiments. Micrographs were taken with a CCD camera (Spot slider; Diagnostic Instruments, Sterling Heights, MI) with a $100 \times$ oil immersion lens (Axiovert; Zeiss, Oberkochen, Germany) at three randomly chosen positions per culture dish. At least two coverslips were analyzed per time point and experimental condition in each of at least two experiments. At each position, one micrograph was taken with the fluorescence filter set optimized for the Cy3 label (filter set, 15; excitation, 546/12; beam splitter, 580; emission, 590 ), and another micrograph was taken with a narrow-band fluorescence filter set optimized for Cy2 (filter set, 13; excitation, 470/20; beam splitter, 493; emission, 505-530; both from Zeiss). The bandwidth of both filter sets was such that no cross talk between the signals of the two antibody stainings occurred. The two micrographs were brought into register using the MetaMorph software (version 5; Universal Imaging Corporation, West Chester, PA), and two to three randomly chosen fields measuring $10 \times 10 \mu \mathrm{m}$ were selected in each pair of photographs. In each field, the amount of all NR1/GluR2/3 (or NR1/synaptophysin) colocalized clusters was determined and expressed as percentage of all NR1-positive clusters. The differences in proportions of double-labeled synaptic clusters were tested by comparing the sum of double-labeled synaptic clusters with the sum of single-labeled synaptic clusters in each condition and time point ( $\chi^{2}$ test). The total numbers of NR1 synapses examined in each case is given in the relevant figure legends.

Axonal outgrowth in culture compartments. Compartment cultures were generated by delimiting plating space until cells adhered to the substrate. Silicon templates were added after poly-D-lysine coating onto the air-dried coverslip. The templates contained three compartments measuring $2 \times 7 \mathrm{~mm}$ each (see Fig. $7 \mathrm{~A}$ ). The distance between the middle compartment (A) and one outer compartment (B) was $1.5 \mathrm{~mm}$, and the distance between the middle compartment (A) and the other outer compartment (C) was $2.0 \mathrm{~mm}$ (see Fig. 7A). At the time of plating, 300 cells $/ \mathrm{mm}^{2}$ were added to each compartment, and, after $2 \mathrm{~h}$, the silicon template was gently removed from the culture, allowing axons to freely interconnect compartments.

To estimate the time point when the first axons reach the neighboring compartment, neurons were plated only into the middle compartment of a silicon template (see Fig. 7A, compartment A) as described above (single-compartment culture). Cultures were fixed at $6,9,12,15,18$, and $21 \mathrm{DIV}$ for $30 \mathrm{~min}$ at $36^{\circ} \mathrm{C}$ in $4 \%$ paraformaldehyde in phosphate buffer $(0.1 \mathrm{M}, \mathrm{pH} 7.4)$ containing $0.12 \mathrm{M}$ glucose, and axons were stained with an antibody for unique $\beta$-tubulin III (TuJ1; Babco, Richmond, CA). After fixation, cultures were washed in PBS and preincubated with $0.25 \%$ Triton X-100 in PBS for $10 \mathrm{~min}$ at room temperature. The subsequent antibody incubations were each followed by several washes with PBS/ monoclonal mouse anti-TuJ1 (1:1000) in 2\% bovine serum albumin, $10 \%$ normal goat serum, $0.3 \%$ Triton X-100, and 5\% sucrose in PBS, overnight at $4^{\circ} \mathrm{C}$, followed by goat anti-mouse Cy3 (1:400; Jackson ImmunoResearch) for $2 \mathrm{~h}$ at room temperature. After the final PBS wash, 
coverslips were dehydrated in an ethanol series, cleared in two changes of xylene, and mounted over clean slides with Fluoromount. For quantitative analysis, the maximal axonal outgrowth distance of the 50 longest axons was determined in each culture with a NeuroLucida system (MicroBrightField, Magdeburg, Germany). The distance was measured by putting a straight line perpendicular to the compartment border from the border to the outermost point that an axon had reached. A total of seven cultures from five preparations were analyzed for each time point. The 50 longest axons of each culture were selected for comparison between time points (Kruskal-Wallis one-way ANOVA on ranks, followed by a pairwise multiple comparison procedure with the Tukey's test).

Dextran tracing of intercompartmental connections. To assess anatomical connections between the compartments, tetramethylrhodamine dextran D-3308 (Molecular Probes, purchased from MoBiTec, Goettingen, Germany) was applied to the middle compartment of cultures where cells had been plated to all three compartments. One milligram of the dye was diluted in $50 \mu \mathrm{l}$ of distilled water. From this stock solution, $5 \mu \mathrm{l}$ were air dried on one side of a $3 \times 3 \times 8 \mathrm{~mm}$ stainless steel block. After carefully injuring the axons at the border of the middle compartment by small scratches with the tip of a scalpel, the dye-coated side of the stainless steal block was carefully placed exactly onto the middle compartment, and the cultures were kept in the incubator for another $16 \mathrm{~h}$. During incubation, the dye was taken up by the injured axons in the middle compartment and transported in retrograde and anterograde directions. After fixation $\left(30 \mathrm{~min}, 36^{\circ} \mathrm{C}, 4 \%\right.$ paraformaldehyde), coverslips were incubated for $1 \mathrm{~min}$ in DAPI $\left(4^{\prime}, 6^{\prime}\right.$-diamidino-2-phenylindole, $50 \mu \mathrm{g} /$ $\mathrm{ml}$; Sigma-Aldrich, Seelze, Germany) to stain cell nuclei. After a final wash in PBS, coverslips were dehydrated in an ethanol series, cleared in two changes of xylene, and mounted on clean slides with Fluoromount. Cell body position of retrograde-labeled neurons were plotted within the two outer compartments with a NeuroLucida system. The density distribution of the labeled neurons was calculated by dividing each $2 \times 7 \mathrm{~mm}$ compartment in parallel $0.5 \times 7 \mathrm{~mm}$ zones. Labeled neurons in each stripe were counted, and results were expressed as distribution histograms of all reconstructed cultures.

Double patch-clamp recordings. Functional connections between compartments were assessed with double patch-clamp recordings in 13- to 16-d-old cultures. An acrylic ring was fixed to the culture dish with silicon grease, resulting in a chamber with a volume of $1-1.5 \mathrm{ml}$. This chamber was mounted to the stage of an inverted microscope and continuously perfused at $1-2 \mathrm{ml} / \mathrm{min}$ with HEPES-buffered Ringer's solution (in mM: $140 \mathrm{NaCl}, 5 \mathrm{KCl}, 1.5 \mathrm{CaCl}_{2}, 0.75 \mathrm{MgCl}_{2}, 1.25 \mathrm{NaH}_{2} \mathrm{PO}_{4}, 20$ D-glucose, and 15 HEPES/NaOH, pH 7.4) (Opitz et al., 2002). The electrophysiological recordings were performed at room temperature. Whole-cell voltage-clamp recordings were performed with an Axoclamp-2B (Axon Instruments, Foster City, CA) and with an EPC-7 (HEKA Elektronik, Darmstadt, Germany) amplifier. Patch pipettes had a tip resistance of 3-5 M $\Omega$ when filled with internal solution (in mM: 115 $\mathrm{CsCl}, 30$ tetraethylammonium-Cl, $2.2 \mathrm{MgCl}_{2}$, and $11 \mathrm{HEPES} / \mathrm{CsOH}, \mathrm{pH}$ 7.3). Cortical networks at that developmental stage (13-16 DIV) typically exhibit synchronous activity that can be monitored in single neurons as barrages of synaptic currents lasting several hundreds of milliseconds (Opitz et al., 2002). Network activity was recorded simultaneously in two neurons at different locations for 10-30 min depending on its frequency. The delay between the onset of the rhythmic network discharge in the two cells was extracted from the continuous recordings using Clampfit 9 (Axon Instruments). The two recording sites were considered as functionally connected when the majority of those delays fell within $250 \mathrm{~ms}$ (see Fig. 9A). Compartments were considered not functionally connected when bursts occurred with independent frequencies (most delays $>1000$ ms between both compartments) (see Fig. 9B).

Calcium imaging. For recording intracellular calcium concentration $\left(\left[\mathrm{Ca}^{2+}\right]_{\mathrm{i}}\right)$ changes in neuronal networks, cell cultures were incubated in $5 \mu \mathrm{M}$ fluo-3 pentacetoxy-methylester (Molecular Probes, purchased from MoBiTec, Goettingen, Germany) for $1 \mathrm{~h}$, followed by several washes with HEPES-buffered Ringer's solution (in mM: $140 \mathrm{NaCl}, 5 \mathrm{KCl}$, $1.5 \mathrm{CaCl}_{2}, 0.75 \mathrm{MgCl}_{2}, 1.25 \mathrm{NaH}_{2} \mathrm{PO}_{4}, 20$ D-glucose, and $15 \mathrm{HEPES} /$ $\mathrm{NaOH}, \mathrm{pH}$ 7.4) (Voigt et al., 2001; Opitz et al., 2002). Sequences of frames were recorded at $1 \mathrm{~Hz}$ from randomly chosen fields on an inverted microscope equipped with a cooled charge-coupled device camera (Princeton Scientific Instruments, Monmouth Junction, NJ). Images were processed with MetaMorph software (version 3.5; Universal Imaging Corporation). A change in $\left[\mathrm{Ca}^{2+}\right]_{\mathrm{i}}$ was considered significant when the absolute difference of gray values exceeded five times the SD of background noise measured in cell-free areas. A neuron was considered active when it showed a significant transient increase in its $\left[\mathrm{Ca}^{2+}\right]_{\mathrm{i}}$ at least once during the recording period. The total number of neurons in the field was obtained from light microscopic differential interference contrast images taken from this field before imaging. The percentage of synchronously active neurons was determined from one frame in a recording session that showed the maximal fraction of all neurons that increase their $\left[\mathrm{Ca}^{2+}\right]_{\mathrm{i}}$ in synchrony (see Figs. 3, 4). The criteria for synchrony was the coherent onset in time of the $\left[\mathrm{Ca}^{2+}\right]_{\mathrm{i}}$ change of the participating neurons as is shown in Figure 3, $A$ and $B$. Nonsynchronous neurons are those neurons that increased their $\left[\mathrm{Ca}^{2+}\right]_{\mathrm{i}}$ at least once during the recording period but did not participate in any synchronous event. In those cases in which the changes in $\left[\mathrm{Ca}^{2+}\right]_{i}$ were recorded in BMI-, APV-, or CNQXtreated cultures, the drugs were also present during Fluo-3 loading, in consecutive washing steps, and during recording. The differences in proportions of synchronous neurons (relative to total number of neurons) were tested by comparing the sum of synchronous neurons with the sum of nonsynchronous neurons in each condition and time point ( $\chi^{2}$ test). The total numbers of neurons examined in each case is given in the relevant figure legends.

\section{Results}

\section{AMPAfication of synapses}

It has been shown that colocalization of NR1 and GluR2/3 receptors occurs mostly within synaptic contact sites (Petralia et al., 1999). With electrophysiological methods, it has also been shown that synapses that contain only NR1 receptors are functionally silent, whereas synapses that contain NR1 and GluR2/3 receptors are functionally activated (Isaac, 2003; Voronin and Cherubini, 2003). To detect the activation of presumptive silent synapses morphologically, we examined the colocalization of AMPA and NMDA receptors in cortical cultures between 6 and 15 DIV using several monoclonal and polyclonal antibodies, as has been done previously (Carroll et al., 1999; Liao et al., 1999, 2001; Petralia et al., 1999). Receptor immunostaining in cortical cultures showed well defined spots of immunoreactivity within the neuropil, which were considered to be clusters of receptors (Fig. 1) (Liao et al., 2001). The quantification of receptor clusters was made relative to the total of NR1 clusters, because this receptor type is present in both silent and activated synapses. Because not all of the stained NR1 receptor clusters are located at synaptic release sites, we first controlled the amount of synaptic NR1 clusters by combining NR1 and synaptophysin immunocytochemical staining (Fig. 1G-I). Assuming that NR1 clusters that are colocalized with GluR2/3 are most probably synaptic (see above), it follows that the proportion of NR1/GluR2/3 clusters will not exceed the proportion of NR1/synaptophysin clusters. Indeed, experiments show that the fraction of NR1/GluR2/3 colocalization matches the fraction of NR1/synaptophysin colocalization in cultures expressing synchronous network activity (Fig. 2A).

In another series of control experiments, we compared the colocalization of NR1 receptors with GluR2/3 and GluR1 subunits (Fig. $2 B$ ). These experiments showed that a staining with NR1 antibodies followed by GluR1 plus GluR2/3 antibodies mixture resulted in the same amount of colocalized NR1/GluR receptor clusters as an antibody staining with NR1 followed by GluR2/3 alone. The double staining of cultures with NR1/GluR1 resulted in significant less amount of colocalized clusters. Because the GluR2/3 antibodies recognize the receptor subunit 2 that is frequently coexpressed with subunit 3 or 1 (Craig et al., 
1993), we concluded from these results that the NR1/GluR2/3 staining included the population of receptors that contain GluR1/2 subunits. Based on these data, we combined in the present experiments either anti-NR1 and anti-GluR2/3 antibodies or anti-NR1 and a mixture of antiGluR2/3 and anti-GluR1 antibodies. In the following experiments, to determine the changes from silent synapses to activated synapses, we expressed the NR1/GluR2/3 clusters counted as a fraction of all NR1 clusters (see Materials and Methods).

\section{Blockade of synchronous activity delays the AMPAfication of synapses}

In the next set of experiments, we tested the hypothesis that one of the functions of synchronous network activity is the collective activation of silent synapses (Figs. 3, $4 A, B)$. We first compared the development of synchronous network activity and NR1/GluR2/3 expression in control cultures. At 6 DIV, cultures were spontaneously active, but synchronous network activity had not yet developed (Fig. 3C, $4 A$, black columns). Between 6 and 9 DIV, synchronous network activity appeared, and, at $9 \mathrm{DIV}, 54 \%$ of the neuronal population participated in synchronous network activity. The fraction of synchronously active neurons increased steadily until the entire network was synchronously active at 15 DIV. The increase in the proportion of participating neurons between 9 and 15 DIV is mostly attributable to cell elimination during this time period, as has been shown previously (Voigt et al., 1997; Opitz et al., 2002). Receptor staining of age-matched sister cultures showed that the proportion of NR1 clusters that were colocalized with GluR2/3 increased accordingly during a similar time interval (6-12 DIV) as synchronous network activity increased (Fig. $4 B$, black column). Starting at $8.5 \%$ at 6 DIV when synchronous network activity had not yet developed, the fraction of NR1/GluR2/3 clusters increased to $30.2 \%$ at 9 DIV when the network expressed synchronous activity. At 12 DIV, the fraction of NR1/GluR2/3 clusters had reached $42.6 \%$, with no additional increase thereafter. These results suggests a parallel development of synchronous network activity and AMPAfication of synapses.

It has been shown recently that AMPAfication of silent synapses is induced by synchronous presynaptic and postsynaptic depolarization (Isaac et al., 1995, 1997; Liao et al., 1995; Rumpel et al., 1998; Feldman et al., 1999), an effect that is mediated via the NMDAR. Because all participating neurons depolarize their membrane potential in synchrony during synchronous network activity (Figs. 3, 4) (Voigt et al., 1997, 2001; Opitz et al., 2002), we hypothesized that this form of population event inevitably leads to the massive AMPAfication of the silent synapses present between coactivated neurons. To test this hypothesis, we delayed the onset of synchronous network activity for several days by chronic application of BMI to the culture medium (Opitz et al., 2002). As shown previously (Voigt et al., 2001), in 8- to 12-d-old cultured neurons, the reversal potential of currents induced by $\mathrm{GABA}_{\mathrm{A}}$ receptor agonist muscimol is more positive than the
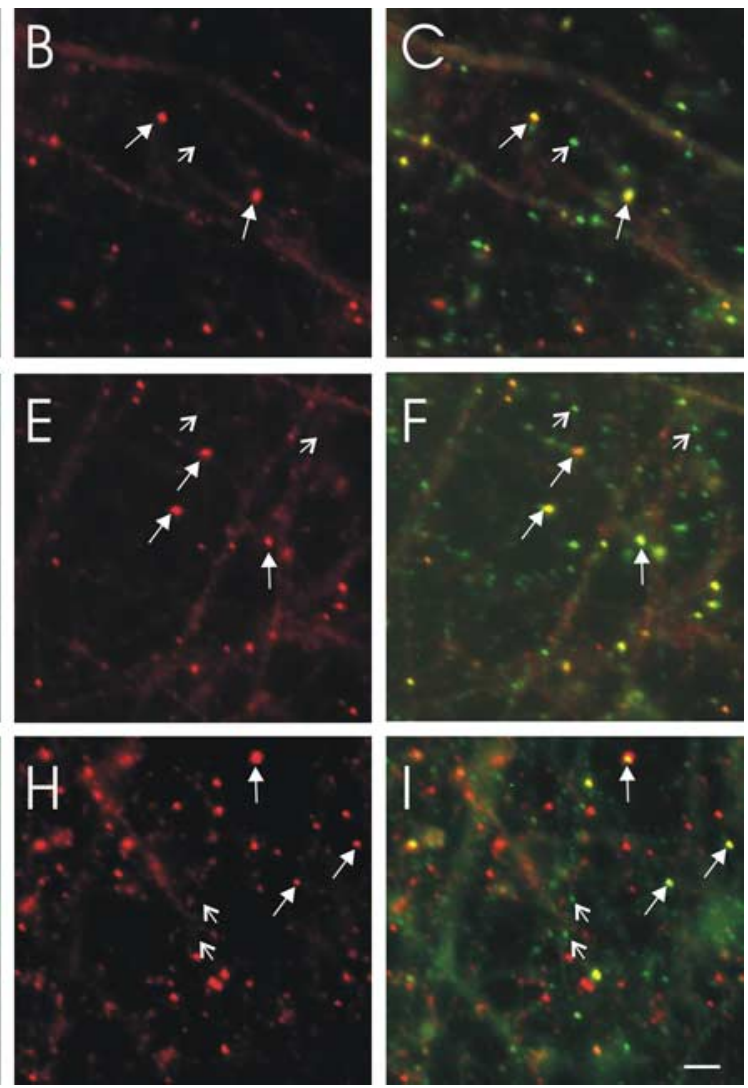

Figure 1. Colocalization of NR1/GluR2/3 (A-F) and NR1/synaptophysin $(\boldsymbol{G}-\boldsymbol{I})$ immunostaining in 9-d-old cultures. Each row images shows double labeling of a different field. Images of immunostained NR1 $(\boldsymbol{A}, \boldsymbol{D}, \boldsymbol{G}), \mathrm{GluR2} / 3(\boldsymbol{B}, \boldsymbol{E})$, and synaptophysin $(\boldsymbol{H})$ clusters were combined $(\boldsymbol{C}, \boldsymbol{F}, \boldsymbol{I})$ to show that some of the NR1-containing clusters are colocalized with GluR2/3containing $(\boldsymbol{C}, \boldsymbol{F})$ or synaptophysin-containing $(\boldsymbol{I})$ clusters (long arrows), whereas others are not (short arrows). Scale bar, $2 \mu \mathrm{m}$.
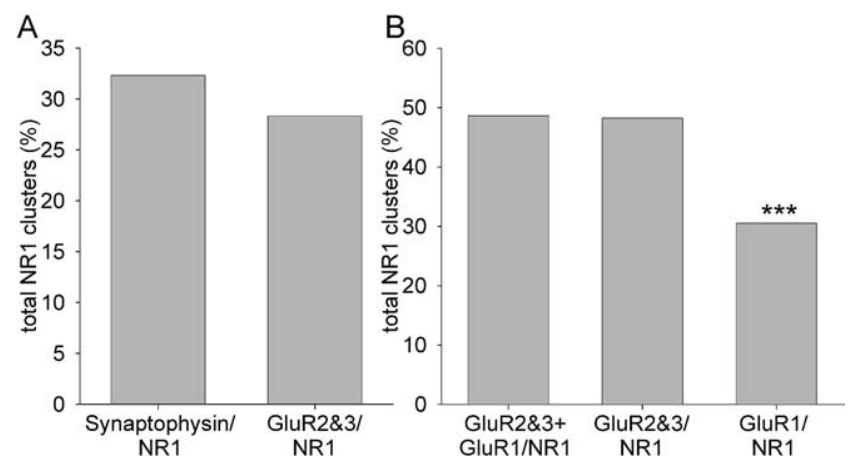

Figure 2. Quantitative analysis of NR1/synaptophysin and NR1/GluR2/3 colocalization in 9-d-old cultures. $\boldsymbol{A}$ shows the relative number of NR1-immunoreactive clusters that contained either synaptophysin or GluR2/3. The percentage of NR1 cluster colocalized with GluR2/3 was statistically not different from the percentage of NR1 clusters colocalized with synaptophysin, suggesting that the majority of NR1/GluR2/3 clusters are synaptic ( $\chi^{2}$ test; 27 analyzed fields from 3 cultures). Total number of NR1 clusters examined for colocalization with GluR2/3, $n=$ 600 ; for colocalization with synaptophysin, $n=724$. $B$, Colocalization of NR1 clusters with clusters stained by antibodies against the GluR2/3 and the GluR1 subunit. Although NR1/ GluR2/3 colocalization is comparable with NR1/GluR1 plus GluR2/3 colocalization, GluR1 colocalizes in a much lower fraction of NR1-containing clusters ( $\chi^{2}$ test; ${ }^{* * *} p \leq 0.001 ; 36$ analyzed fields from 4 cultures; total number of NR1 clusters examined, $n=683$ for NR1/GluR2/3, $n=$ 616 for NR1/GluR1 plus GluR2/3, and $n=567$ for NR1/GluR1).

measured resting membrane potential. During this time window, $\mathrm{GABA}_{\mathrm{A}}$ stimulation (in the presence of glutamate receptor antagonists) evokes a calcium transient in all cells under investigation. Moreover, in these young cultures, $\mathrm{GABA}_{\mathrm{A}}$ blockade inhibits 

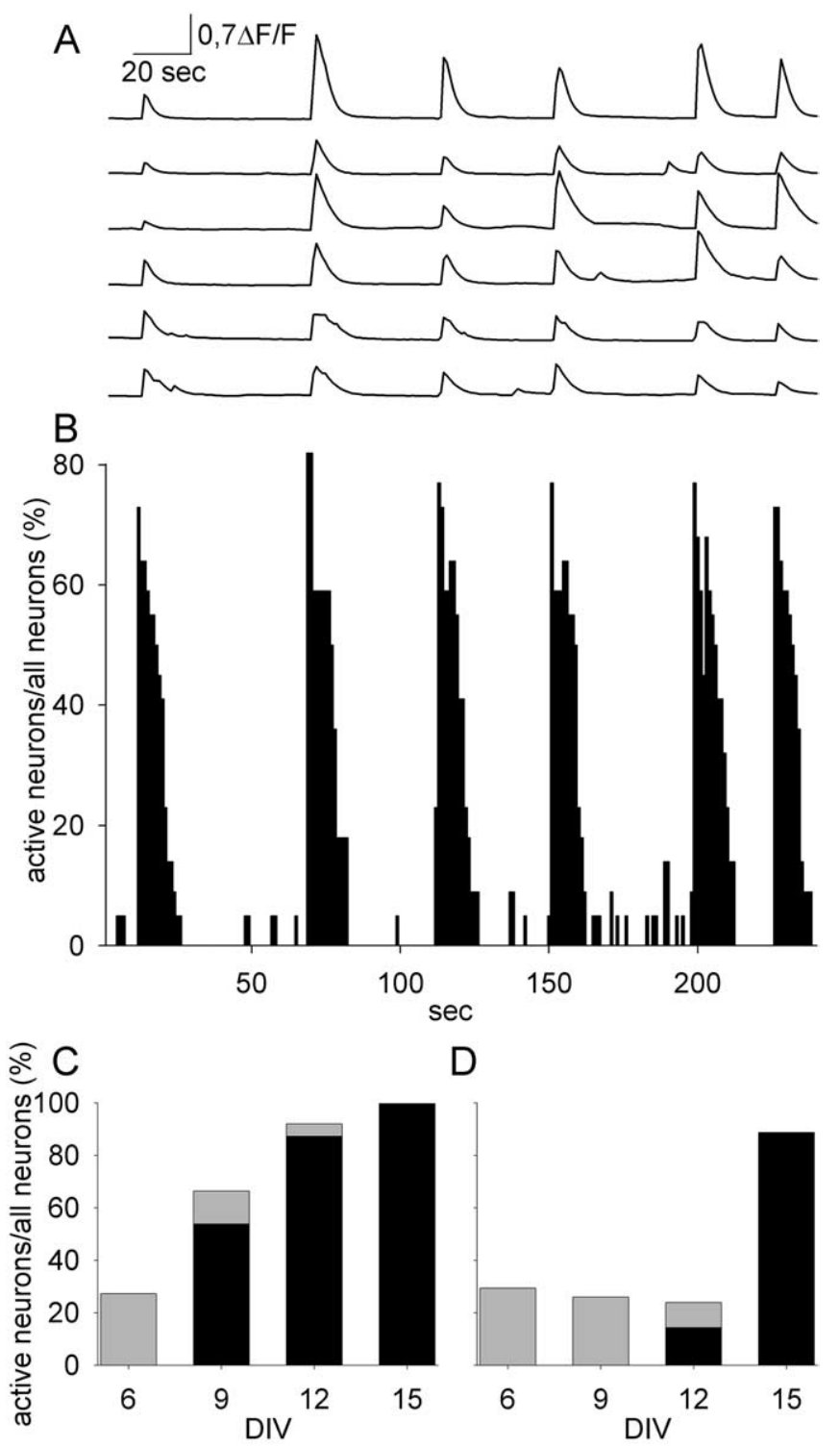

synchron neurons/all neurons (\%)

non synchronous neurons/all neurons (\%)

Figure 3. Spontaneous synchronous $\left[\mathrm{Ca}^{2+}\right]_{i}$ oscillations in cultured neocortical neurons. $A$, Fluorometric $\mathrm{Ca}^{2+}$ recordings of synchronous $\left[\mathrm{Ca}^{2+}\right]_{i}$ oscillations of six neurons in a $12-\mathrm{d}$-old culture. $B$, Activity histogram of all neurons present in the same field as cells shown in $A$. During this recording, a maximum of 18 of 22 neurons showed a synchronous increase in their $\left[\mathrm{Ca}^{2+}\right]_{\text {. }}$. C, Development of network activity in a control culture, showing the percentage of synchronous and nonsynchronous neurons (nonsynchronous neurons showed changes in $\left[\mathrm{Ca}^{2+}\right]_{\mathrm{i}}$ during the recording period but did not participate in synchronous events). $\boldsymbol{D}$, Development of network activity in cultures raised in the presence of the $G_{A B A_{A}} R$ antagonist $B M I$ (drug added at 5 DIV).

spontaneous network activity, independent of glutamatergic activity (Voigt et al., 2001; Opitz et al., 2002).

In the presence of BMI, synchronous network activity was significantly lower than control until 12 DIV and then increased to nearly control values at $15 \mathrm{DIV}$ (Figs. 3D, 4A, light gray bars). Note, however, that a gradual increase in synchronous spontaneous activity did occur under chronic BMI treatment. Correspondingly, the fraction of NR1/GluR2/3 receptor clusters increased, while remaining significantly lower compared with control cultures. The fraction of NR1/GluR2/3 receptor clusters was comparable with control values when synchronous activity ratio had reached control values at 15 DIV (Fig. $4 B$ ).
To further test the hypothesis, we increased the spontaneous activity of the cultures by lowering the $\left[\mathrm{Mg}^{2+}\right]$ of culture medium. The decrease of $\left[\mathrm{Mg}^{2+}\right]$ in the culture medium removes the magnesium block from the NMDA channels and, as a consequence, glutamate may activate NMDA-containing synapses without the need of postsynaptic depolarization. Additionally, this experiment would also give an estimate of the maximal number of NR1/GluR2/3 synapses that could be activated at a given time point (Fig. 4A, $B$ dark gray columns). The stimulation was performed in both untreated and BMI-treated sister cultures after removal of BMI. In all cases, the fraction of synchronous active neurons as well as the fraction of NR1/GluR2/3 colocalized clusters did not significantly differ $\left(\chi^{2}\right.$ test $)$. For this reason, the data of both groups (stimulated untreated control cultures and stimulated BMI-treated cultures) were pooled. At 6 DIV, 0 mM $\mathrm{Mg}^{2+}$ stimulation elicited synchronous activity in $29.1 \%$ of the neurons (Fig. $4 A$ ) compared with $0 \%$ in control. The immunocytochemical analysis of receptor expression showed after stimulation that the activation of synchronous network activity dramatically increased the fraction of NR1/GluR2/3 clusters (Fig. $4 B$ ). This indicated that, in 6 DIV cultures, a pool of silent synapses existed ready to be activated by synchronous network activity. After $6 \mathrm{DIV}$, the $0 \mathrm{~mm} \mathrm{Mg}{ }^{2+}$ stimulation was not able to increase the fraction of $\mathrm{NR} 1 / \mathrm{GluR} 2 / 3$ receptor clusters compared with control (Fig. $4 \mathrm{~B}$ ), indicating that, under control conditions, synchronous network activity was capable of activating the majority of silent synapses.

To exclude the possibility that changes in receptor colocalization were the result of an activity-dependent change in the total amount of NR1-containing synapses, we determined the fraction of NR1/synaptophysin and NR1/GluR2/3 clusters in 9-d-old sister cultures grown under control conditions, in the presence of BMI, and after $0 \mathrm{mM} \mathrm{Mg}^{2+}$ stimulation (see above). Whereas the fraction of synapses containing NR1 receptors was independent of the network activity, the fraction of NR1/GluR2/3-containing synapses was significantly reduced when synchronous network activity was blocked by BMI (Fig. 4C). Thus, activity did not modulate the overall synaptic content but did change the number of activated synapses.

From the results above, we concluded that decreasing synchronous spontaneous activity by blocking $\mathrm{GABA}_{\mathrm{A}}$ function decreased the probability of activating silent synapses. Conversely, the increase in NMDA depolarization by lowering $\left[\mathrm{Mg}^{2+}\right]$ dramatically increased network synchronization and concurrently activated silent synapses. Although a single coincidence between presynaptic and postsynaptic activity might be sufficient to activate a single silent synapse, global synchronous network activity might be advantageous for the simultaneous AMPAfication of a population of immature synapses.

\section{Time course of silent synapse activation}

To investigate the time course of the synaptic activation by synchronous network activity, cultures were raised in BMI until the ninth day in vitro, transferred to a $0 \mathrm{mM} \mathrm{Mg}^{2+}$ medium, and examined after increasing time intervals (Fig. 5A). Already after 15 min of stimulation (first time point investigated), a significant increase in the fraction of NR1/GluR2/3-containing clusters was observed compared with unstimulated BMI cultures. After 30 min, the fraction of NR1/GluR2/3-containing clusters was not different from control. These data show that, as soon as the network becomes synchronously active, GluR2/3 receptors become incorporated within minutes and the synapses become activated. By adding CNQX or APV to the $0 \mathrm{~mm} \mathrm{Mg}^{2+}$ medium, we asked 

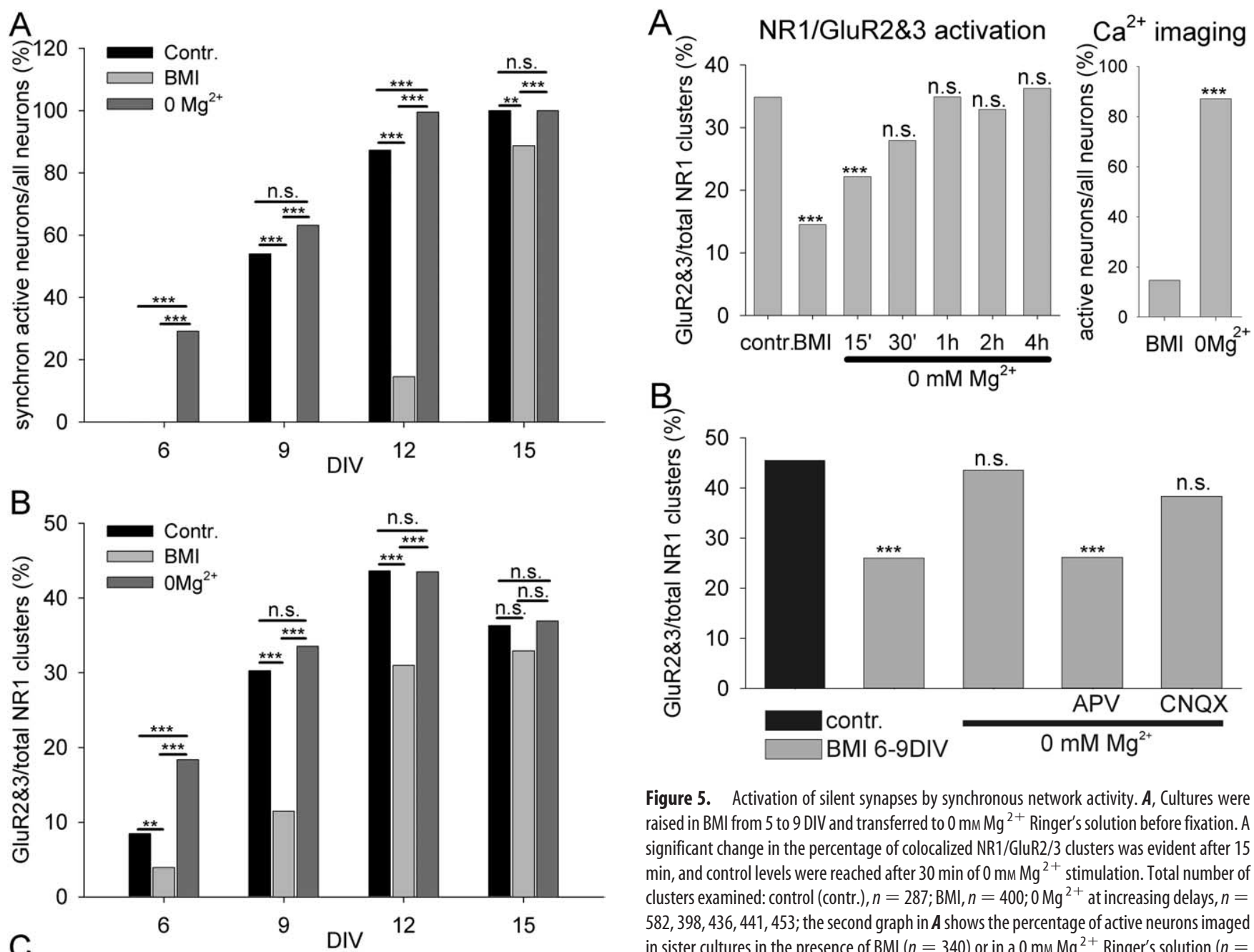

Figure 5. Activation of silent synapses by synchronous network activity. $A$, Cultures were raised in BMI from 5 to 9 DIV and transferred to $0 \mathrm{~mm} \mathrm{Mg}^{2+}$ Ringer's solution before fixation. A significant change in the percentage of colocalized NR1/GluR2/3 clusters was evident after 15 $\mathrm{min}$, and control levels were reached after $30 \mathrm{~min}$ of $0 \mathrm{~mm} \mathrm{Mg}{ }^{2+}$ stimulation. Total number of clusters examined: control (contr.), $n=287 ; \mathrm{BMl}, n=400 ; 0 \mathrm{Mg}^{2+}$ at increasing delays, $n=$ $582,398,436,441,453$; the second graph in $\boldsymbol{A}$ shows the percentage of active neurons imaged in sister cultures in the presence of BMI $(n=340)$ or in a $0 \mathrm{mM} \mathrm{Mg}^{2+}$ Ringer's solution $(n=$ 478). Although the 9-d-old cultures presented no synchronicity between the spontaneously active neurons under BMI, strong synchronized activity occurred under $0 \mathrm{~mm} \mathrm{Mg}^{2+}$ stimulation. $B$, The fraction of NR1/GluR2/3 clusters was determined in 9 DIV cultures cultivated in the presence of the $\mathrm{GABA}_{A}$ receptor antagonist BMI (gray columns; $n=667$ ) and compared with control cultures (black column; $n=556$ ). Three hours before fixation, three sets of cultures were transferred to $0 \mathrm{~mm} \mathrm{Mg}{ }^{2+}$ Ringer's solution (third column; $n=519$ ) or to $0 \mathrm{~mm} \mathrm{Mg}^{2+}$ Ringer's solution containing either the NMDA receptor antagonist APV (fourth column; $n=$ 561 ) or the AMPA receptor antagonist CNQX (fifth column; $n=669$ ). Unstimulated cultures raised in the presence of $B M I$ (no synchronous network activity) or stimulated cultures in the presence of APV (blocked NMDA receptors) showed a significantly lower fraction of NR1/ GluR2/3 clusters compared with control cultures. Conversely, the fraction of NR1/GluR2/3 clusters in cultures raised in BMI and stimulated with $0 \mathrm{~mm} \mathrm{Mg}^{2+}$ in either the absence or presence of CNQX before fixation did not differ significantly from control cultures. The stimulation in $\boldsymbol{A}$ and $\boldsymbol{B}$ was performed in BMI-treated cultures after removal of BMI ${ }^{* * *} p \leq 0.001 ; \chi^{2}$ test; 18 analyzed fields from 2 cultures per time point in all cases; for imaging, 5 fields from 1 culture were analyzed per time point). n.s., Not significant.

contr.

$\mathrm{OMg}$

Synaptophysin/NR1 GluR2\&3/NR1

Figure 4. Coexpression of NR1/GluR2/3 correlates with synchronized network activity between 6 and 15 DIV in three experimental conditions. $\boldsymbol{A}$, In untreated control cultures, the percentage of simultaneously active neurons increased gradually between 6 and 15 DIV (black bars). Under BMI, the onset of synchronized activity was delayed until 15 DIV (light gray bars), whereas a $0 \mathrm{mM} \mathrm{Mg}{ }^{2+}$ Ringer's solution increased the percentage of simultaneously active neurons between 6 and 12 DIV but not thereafter (medium gray bars) ${ }^{* *} p=0.005 ;{ }^{* * *} p \leq$ $0.001 ; \chi^{2}$ test; 5 analyzed fields from 2 cultures per time point; total number of cells examined in successive time points: control, $n=280,114,143,135 ; \mathrm{BMI}, n=312,218,129,136 ; 0 \mathrm{~mm}$ $\left.\mathrm{Mg}^{2+}, n=141,127,176,114\right)$. $\boldsymbol{B}$, The percentage of colocalized NR1/GluR2/3 clusters increased in control cultures between 6 and 12 DIV (black bars). This increase was delayed by BMI

treatment (light gray bars) and could be boosted by $0 \mathrm{~mm} \mathrm{Mg}^{2+}$ stimulation (medium gray bars) ${ }^{* *} p=0.028 ;{ }^{* * *} p \leq 0.001 ; \chi^{2}$ test; 18 analyzed fields from 3 cultures per time point; total number of clusters examined in successive time points: control, $n=271,318,199,216$; BMI, $\left.n=318,449,301,259 ; 0 \mathrm{~mm} \mathrm{Mg}^{2+}, n=338,319,204,277\right)$. C, Comparison of NR1/ synaptophysin and NR1/GluR2/3-containing clusters in 9 DIV cultures under control condition (Contr.), in cultures raised in the presence of $\mathrm{BMI}$, and after $0 \mathrm{~mm} \mathrm{Mg}^{2+}$ stimulation. Although there was no significant difference between the NR1/Glu2/3 and NR1/synaptophysin colocalization in control cultures and $0 \mathrm{~mm} \mathrm{Mg}^{2+}$-stimulated cultures, there was a significant difference between NR1/synaptophysin- and NR1/GluR2/3-containing clusters in BMI-treated cultures $\left({ }^{* * *} p \leq 0.001 ; \chi^{2}\right.$ test; 27 analyzed fields from 3 cultures, $9 \mathrm{DIV}$ ) (total number of clusters examined: NR1/synaptophysin, control, $n=724 ; \mathrm{BMI}, n=591 ; 0 \mathrm{Mg}^{2+}, n=685$; NR1/ GluR2/3, control, $\left.n=600 ; \mathrm{BMI}, n=638 ; 0 \mathrm{Mg}^{2+}, n=655\right)$. n.S., Not significant. 

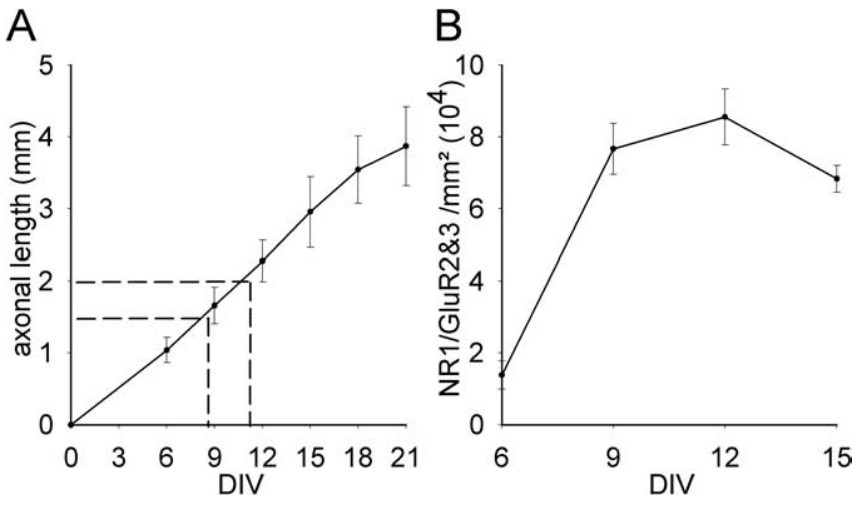

Figure 6. Comparison between axonal outgrowth and synaptic activation time. $\boldsymbol{A}$ shows the maximal distance that individual axons had grown after a given time in culture. To determine the axonal growth rate, neurons were plated into a single compartment, and the straight distance from compartment border to the 50 longest axons was determined in seven cultures ( $n=350$; mean \pm SEM). At approximately 8 DIV, the axons spanned a distance of $1.5 \mathrm{~mm}$ and needed additional $3 \mathrm{~d}$ to reach $2.0 \mathrm{~mm}$ distance from the border. $\boldsymbol{B}$, The density of NR1/GluR2/ 3 -containing clusters increased dramatically between 6 and 9 DIV ( $p \leq 0.001)$, and no significant change occurred thereafter [Kruskal-Wallis one-way ANOVA on ranks, followed by a pairwise multiple comparison procedure (Tukey's test); 18 analyzed fields from 3 cultures per time point].

whether the activation of AMPA or NMDA receptors are necessary for the synaptic activation (Fig. $5 B$ ). The fraction of NR1/ GluR2/3 clusters was determined $3 \mathrm{~h}$ after removal of BMI and transfer to stimulation medium with or without antagonists (Fig. $5 B)$. As expected, this activation can be blocked by the NMDA antagonist APV but not with the AMPA receptor antagonist CNQX (Fig. 5B), confirming that activation of NMDA receptors is required, as has been shown previously (Isaac et al., 1997; Rumpel et al., 1998). After activation of silent synapses, BMI was no longer capable of blocking the synchronous network activity. This indicated that a critical amount of activated glutamatergic synapses is sufficient to generate synchronous network activity in the absence of GABA activation (see above) (Fig. $4 A$ ).

\section{Time constraints for the formation of connections between culture compartments is related to the onset of synchronous activity and the maturation of synapses}

To investigate how the generalized activation of silent synapses may influence the formation of cortical connections, we raised cultures with differently spaced compartments. The rationale was that, if compartments were placed close to each other, the axons might reach their target area before the onset of synchronous network activity and before the activation of silent synapses. If compartments were placed farther away from each other, the axons might reach the target area after the onset of synchronous network activity and after global activation of synapses.

The optimal distance to separate compartments was calculated by measuring the axonal outgrowth in single compartments. To do so, neurons were plated into a single $7 \times 2 \mathrm{~mm}$ compartment, and the straight distance from the tips of the longest axons to the compartment border was measured over time. The axonal outgrowth curve showed an almost linear growth rate up to a distance of $4 \mathrm{~mm}$ (Fig. 6A). The dotted lines in Figure $6 \mathrm{~A}$ show that, between 8 and 9 DIV, i.e., at the time of the onset of synchronous network activity, the axons bridged the distance of $1.5 \mathrm{~mm}$. The distance of $2.0 \mathrm{~mm}$ was not reached before $11 \mathrm{DIV}$, i.e., well after the onset of synchronous network activity (Fig. 4A) and the major increase in activated synapses that occurred between 6 and 9 DIV (Figs. $4 B, 6 B$ ).

Based on these data, compartment cultures were produced in which the distance between compartments $\mathrm{A}$ and $\mathrm{B}$ was $1.5 \mathrm{~mm}$ and between compartments $\mathrm{A}$ and $\mathrm{C}$ was $2.0 \mathrm{~mm}$ (Fig. $7 \mathrm{~A}$ ). The connectivity between these compartments was assessed after the onset of synchronous network activity by either anatomically retrograde and anterograde staining of the axonal connections from the middle compartment to the outer compartments (Figs. 7, 8) or physiologically by double recordings (Fig. 9).

The axonal connections between the compartments were analyzed by application of rhodamine-labeled dextran for $16 \mathrm{~h}$ to the central compartment of 14-17 DIV cultures. After fixation, the number and position of retrogradely labeled neurons were analyzed in the two outer compartments (Figs. 7, 8). The rhodamine-labeled tracer was taken up by injured axons and then transported in anterograde and retrograde directions. Well labeled axons were stained up to the growth cones, whereas neurons showed a strong staining within the soma and the proximal portions of the dendrites (Fig. $7 B, C$ ). The dye stained axons and cell bodies over distances of up to $4 \mathrm{~mm}$. For evaluation of the connectivity between the compartments, only labeled cell bodies were analyzed because axons were more difficult to quantify because of their bifurcations. All 29 successfully dextran-stained cultures showed a similar connectivity pattern between compartments. Ten cultures (four control cultures and six BMI-treated cultures) were used for a completely topographical reconstruction of retrograde-labeled neurons in the two outer compartments (Fig. 8). The distribution of all stained neurons was plotted as histograms showing the density distribution along the short axis of the compartment. The histogram of the four complete analyzed control cultures show that compartment B $(1.5 \mathrm{~mm}$ apart) had a high number of stained cell bodies $(n=947)$, with the highest density of retrograde-labeled neurons at the side facing the central compartment and decreasing density toward the opposite side. Contrary to compartment B, compartment C (2.0 mm apart) had a much lower number $(n=46)$ of retrogradely labeled neurons in all investigated cultures. Thus, connections were established when axons reached their target area before the onset of synchronous network activity but failed to form when the axons reached the target area after the onset of synchronous network activity. This failure to form connections was not based on an alteration in axonal outgrowth capability between compartment $\mathrm{B}$ and $\mathrm{C}$ axons as was verified by measuring the outgrowth distance of compartment $\mathrm{C}$ axons on the side facing away from compartment A. In all 14 DIV cultures of this series $(n=4)$, the mean outgrowth length of the 50 longest axons was $2.53 \mathrm{~mm}$, which fits well with the outgrowth values determined in the single-compartment experiments (Fig. 6). Thus, axons between compartment $\mathrm{B}$ and $\mathrm{C}$ were able to bridge this distance after 14 DIV but failed to form anatomical connections. To test whether the asynchronous onset of synchronous network activity in the two compartments was responsible for this failure, the onset of synchronous activity was delayed by chronic application of BMI. In this case, compartment $\mathrm{A}$ to $\mathrm{C}$ axons had the chance to reach their target area before the AMPAfication of silent synapses, and, in these experiments, a high amount of retrograde-labeled neurons were found in both compartment $\mathrm{B}(1.5 \mathrm{~mm})$ and compartment C (2.0 mm) (B', 792 stained neurons; $C^{\prime}, 439$ stained neurons) (Fig. 8). The high amount of degenerating axons (data not shown) found in control cultures between A to C compartments suggest that axons reached their target area but degenerated when the formation of functional connections failed. 
To functionally assess the degree of connectivity between two compartments, paired patch-clamp recordings were performed. Synchronous network activity elicits a barrage of postsynaptic potentials within the recorded cell (Opitz et al., 2002). If neurons of a simultaneously recorded pair resided within the same compartment but several millimeters apart, the delay between the onset of the population discharge was $<250 \mathrm{~ms}$ (Fig. 9A,C). The same was found if single neurons of a pair were situated in two different but interconnected compartments (Fig. $9 D)$. If the axonal connections between two compartments were interrupted (cut by a scratch with a glass needle), the synchronous network events occurred independently in the two networks, and delays $>1 \mathrm{~s}$ were recorded (Fig. 9B,E). Based on these criteria, we considered two networks as being connected if synchronous events occurred in both recorded neurons within $250 \mathrm{~ms}$ and as not connected if the events occurred with delays $>1$ s. A total of 16 pairs of networks were recorded between 14 and 18 DIV with a distance of $1.5 \mathrm{~mm}$ apart. All of them were interconnected and behaved like a single network (Fig. 9D). From the 25 network pairs separated by $2 \mathrm{~mm}$, only five were connected (Fig. 9F, G).

In a last set of experiments, we assessed directly whether the onset of synchronous network activity (and thereby AMPAfication of glutamatergic synapses) prevents the formation of functional connections between compartments $2 \mathrm{~mm}$ apart. To do so, we chronically applied BMI to the cultures. From the total of 17 pairs of compartments that were recorded between 14 and 18 DIV with a distance of $2.0 \mathrm{~mm}$ apart, we found only one to be connected. Because in the presence of BMI the activation of synapses occurred slowly over days (Fig. 4B) and most likely with different rates in different networks, this failure to show connections between the distant compartments could be related to the uncontrolled timing of activation of the networks in BMI-treated cultures. To ensure a more precise timing, we blocked both GABAergic and glutamatergic synaptic transmission in compartment cultures by applying a mixture of ionotropic glutamate receptor and $\mathrm{GABA}_{\mathrm{A}}$ receptor antagonists (APV plus CNQX plus BMI plus PTX) from 5 to 13 DIV. At day 13, the block was removed by a complete change of the medium, and cultures were investigated at $14-16$ DIV. In all experiments $(n=8)$, pairs of recorded neurons showed connections between compartment $\mathrm{A}$ and $\mathrm{C}$ (Fig. 9H). Thus, experiments strongly suggest that the precise timing between the onset of synchronous network activity and the arrival of connecting axons can either unify or segregate local networks.

\section{Discussion}

The results presented in this paper suggest that the initiation of synchronous network activity in cultured neocortical neurons,
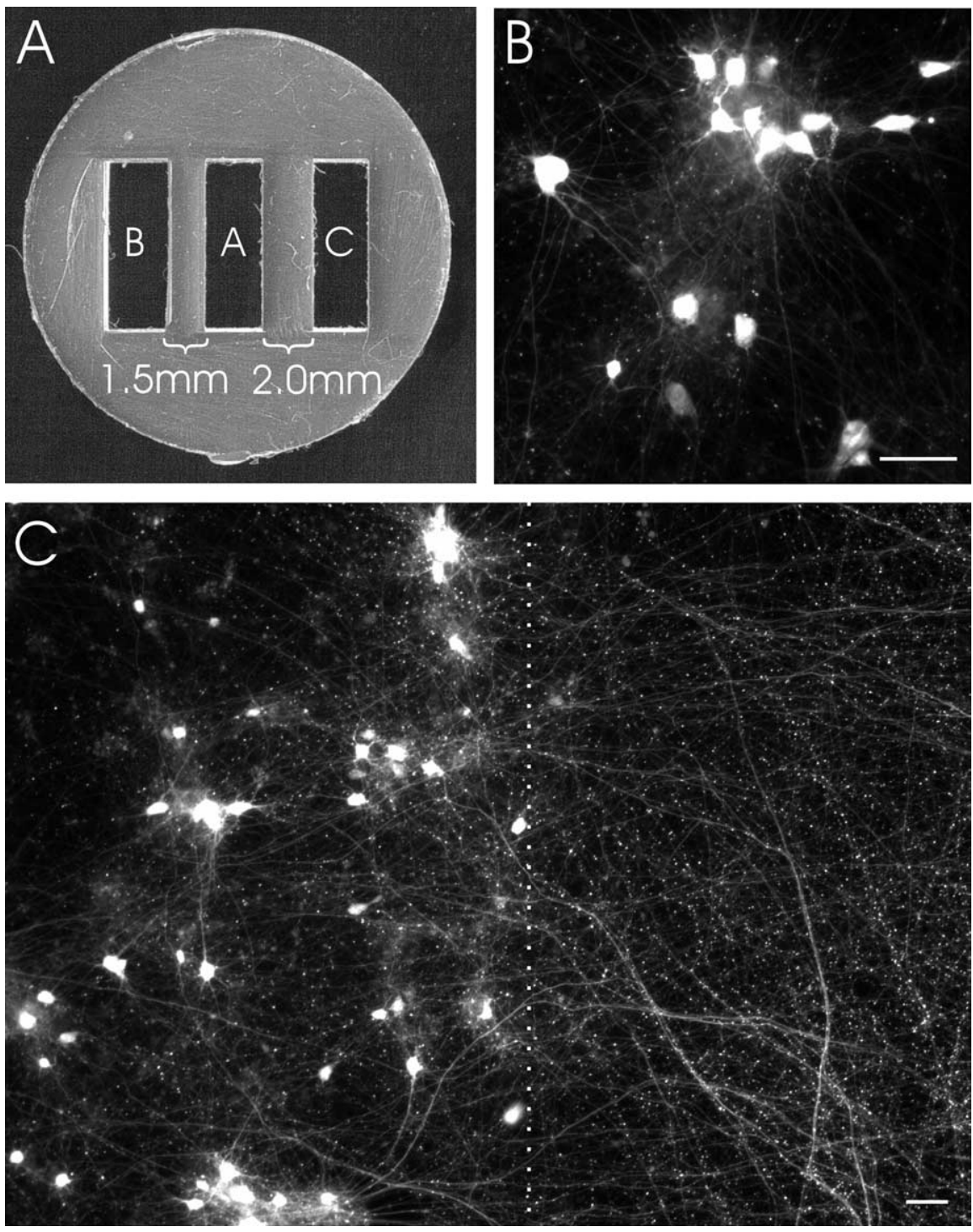

Figure 7. Development of connectivity between compartment cultures. $A$, Silicon template used for plating dissociated cells in defined spaced compartments. Each compartment had a size of $2 \times 7 \mathrm{~mm}$. The distance between compartments $A$ and $B$ was 1.5 $\mathrm{mm}$, and the distance between $A$ and $C$ was $2.0 \mathrm{~mm}$. After the cells had attached to the substrate, the silicon was removed from ments were established. $\boldsymbol{B}$, Examples of neurons in compartment $B$ that were stained by application of a dextran-coated stainless steal block onto compartment A. $C$, The ingrowing axons (left side) and the labeled cell bodies stained by dye application to compartment $A$ are visible in a border field of compartment $B$ (dashed line). Scale bars, $50 \mu \mathrm{m}$.

which depends on basket-like, L-type GABAergic neurons (Voigt et al., 2001; Opitz et al., 2002), consolidates the architecture of axonal connections, most probably by transforming silent to active synapses throughout the network within minutes.

\section{Simultaneous activation of early silent synapses by spontaneous synchronous network activity}

The first evidence for glutamatergic synapses that have NMDARmediated synaptic transmission in the absence of an AMPA receptor-mediated response came from physiological studies that used minimal stimulation protocols (Isaac et al., 1995; Liao et al., 1995). These NMDAR-only synapses have no synaptic response at hyperpolarized potentials but show transmission at depolarized potentials when the $\mathrm{Mg}^{2+}$ block is relieved. Under physiological conditions, this is fullfilled when the presynaptic and postsynaptic neurons are depolarized simultaneously (in syn- 

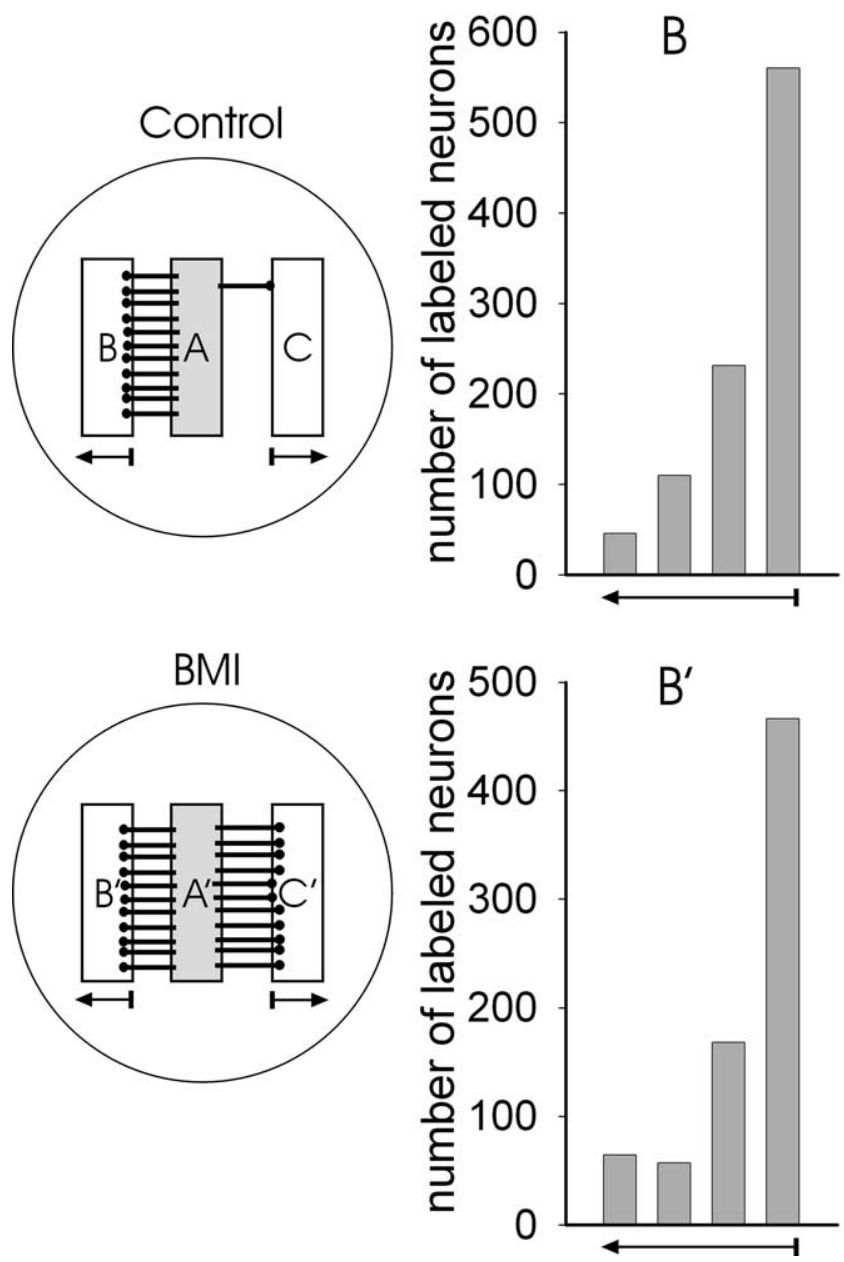

Figure 8. Distribution of anatomical connections between different treated compartment cultures. The top inset (control) shows schematically the distribution of stained neurons under control condition. Dextran was applied to compartment A at 14-17 DIV. Histograms $\boldsymbol{B}$ and $\boldsymbol{C}$ show results from 10 completely reconstructed cultures. Each bin corresponds to the labeled neurons counted within $500-\mu \mathrm{m}$-wide stripes running parallel to the compartment long axis. Topographical order of histogram bins is indicated by the arrows below the $x$-axis. The origin of arrows indicates the border facing compartment $A$ (see inset). In all $B$ compartments (separated by a $1.5 \mathrm{~mm}$ gap from the A compartment), a high amount of neurons were consistently labeled (total 947). In ( compartments (separated by a $2.0 \mathrm{~mm}$ gap from the A compartment), the number of labeled neurons was consistently very low (total 46). The bottom inset (BMI) shows schematically the distribution of stained neurons in cultures grown in the presence of the $G_{A B A} R$ antagonist BMI from 5 to 15 DIV. In these cases, the number of labeled neurons was much higher in compartment C' (total 439) compared with the control situation (C). (Number of neurons labeled in compartment B' was 792 .)

chrony) (Isaac et al., 1995, 1997; Liao et al., 1995; Rumpel et al., 1998; Feldman et al., 1999). Anatomically, the silent synapses possess NMDA receptors but lack AMPA receptors (Durand et al., 1996; Wu et al., 1996; Isaac et al., 1997; Bardoni et al., 1998; Gomperts et al., 1998; Hsia et al., 1998; Li and Zhuo, 1998; Petralia et al., 1999). A synchronous presynaptic and postsynaptic depolarization leads to the AMPAfication of these synapses within minutes (Liao et al., 2001).

In this study, we assessed silent and activated synapses by determining the fraction of NR1 clusters that contained GluR receptors. Because the anti-NR1 antibody recognizes both the surface and the internal receptor pool, only a fraction of the NR1-only clusters were synaptic. In rat hippocampal neurons, it has been reported that $40-50 \%$ of the total NR1 receptors were found on the cell surface (Hall and Soderling, 1997). In our study, we found that $30 \%$ of the NR1 receptor clusters colocalized with synaptophysin. Assuming that synaptophysin is labeling $100 \%$ of the synaptic complexes, this percentage of NR1 colocalized with
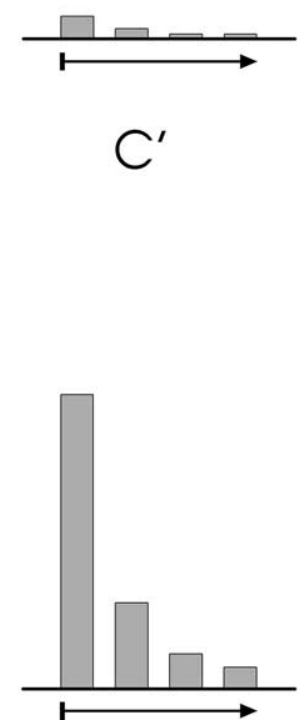

the presynaptic marker corresponds to synaptic NMDAR clusters, and the rest NR1 is most probably part of an extrasynaptic NMDAR pool. This view is supported by the fact that, in sister cultures, the fraction of GluR2/3-containing NR1 clusters did not exceed the fraction of synaptophysin-containing NR1 clusters (Fig. $2 A$ ), indicating that NR/GluR colocalization was synaptic, as has been shown with electron microscopy (Petralia et al., 1999). The results obtained with our evaluation is compatible with the results of other studies using higher-density cortical cultures (Liao et al., 2001). In addition, our results indicate that there is no change in the amount of synaptic NR1 clusters with alterations of activity levels at 9 DIV (Fig. 4C). This is in accordance with studies that show little changes in the NMDA receptor number during AMPAfication and over time (Petralia et al., 1999) or during long-term depression induction (Carroll et al., 1999). Based on these data, it is possible that the $30-50 \%$ of GluR2/3containing NR1 clusters observed in our cultures (Fig. 2A,B) might correspond to $90-100 \%$ of synaptically located NR1 clusters reported on living hippocampal neurons (Pickard et al., 2000).

In our cultures, a maximum of AMPA/ NMDA synapses (relative to total NMDA synapses) is reached at approximately 12 DIV, a time when synchronous network activity becomes independent from GABA $_{\mathrm{A}} \mathrm{R}$ activity (Voigt et al., 2001; Opitz et al., 2002). We show in this paper that spontaneous synchronous activity developing during maturation of the neuronal network is capable of activating the population of silent synapses formed in the initial phase of network formation. The blockade of $\mathrm{GABA}_{\mathrm{A}} \mathrm{R}$ transmission, an intervention that does not interfere with the development of glutamatergic transmission, delays the onset of synchronous network activity (Voigt et al., 2001; Opitz et al., 2002) and also delays the activation of silent synapses. Conversely, the stimulation of the network (by lowering extracellular $\left[\mathrm{Mg}^{2+}\right]$ ) activates silent synapses within minutes (Fig. 5).

From the experiments above, we suggest that one function of synchronous network activity is the global activation of early silent synapses, a process that contributes to the consolidation of intrinsic connections during early brain development.

\section{The appearance of synchronous network activity limits the range of corticocortical connections}

Because synchronous activity occurs across the whole neonatal neocortex, it has been suggested that it might be involved in the development of long range cortical connections (Garaschuk et al., 2000). A link between synchronous activity and the establishment of functional axonal connections may be provided by the 
effect of early synchronous activity on immature glutamatergic synapses.

To test this hypothesis, we designed compartment cultures that expose outgrowing axons to two types of situations. In one situation, the outgrowing axons reach the adjacent compartment shortly before the onset of synchronous network activity, i.e., before the onset of global activation of silent synapses; in the other situation, the axons reach the adjacent compartment shortly after the onset of synchronous network activity. The outcome of these experiments was that compartments connected well when the axons reached their target area before the onset of synchronous network activity and failed to connect when the axons reached the neighboring compartment after the onset of synchronous network activity (Figs. 7-9). Limited growth capacity of axons could not explain these differences because axons can grow for much longer distances (Fig. 6A). In well connected A to B compartments $(1.5 \mathrm{~mm})$, dextran-labeled axons frequently traversed the entire compartment B, reaching a length of at least $3.5 \mathrm{~mm}$ from its origin in compartment A. In addition, in those 14-d-old cultures in which no connections had developed between compartments A and $\mathrm{C}(2.0 \mathrm{~mm})$, the axons from neurons in compartment $\mathrm{C}$ had grown out for over $2.53 \mathrm{~mm}$ on the side facing away from the compartment A. In some of the cases (5 of 25), compartments separated by $2.0 \mathrm{~mm}$ developed connections, possibly because in these cases some fast-growing axons reached the target area before the onset of synchronous network activity. The strongest argument to show that distance is not the limiting factor for axonal growth comes from experiments in which the onset of synchronous network activity was delayed for several days by blocking glutamatergic and GABAergic transmission. In all of these cases, compartments established functional connections (Figs. 8, 9). The fact that a high amount of degenerating axons was routinely found between nonconnected compartments indicates that, in control cultures, axons had reached their target area but subsequently degenerated (data not shown).

How to explain the failure of axons to innervate an adjacent compartment after the onset of synchronous network activity? An explanation would be that network activity developing in separated compartments prevents innervation because connections between neurons that are predominantly activated out-of-phase are eliminated. If network activity includes all neurons in a compartment, each neuron will most probably depolarize during the compartment events and may not or only rarely depolarize by the asynchronous activity coming through pioneer axons from the other compartment, thus ex-
A
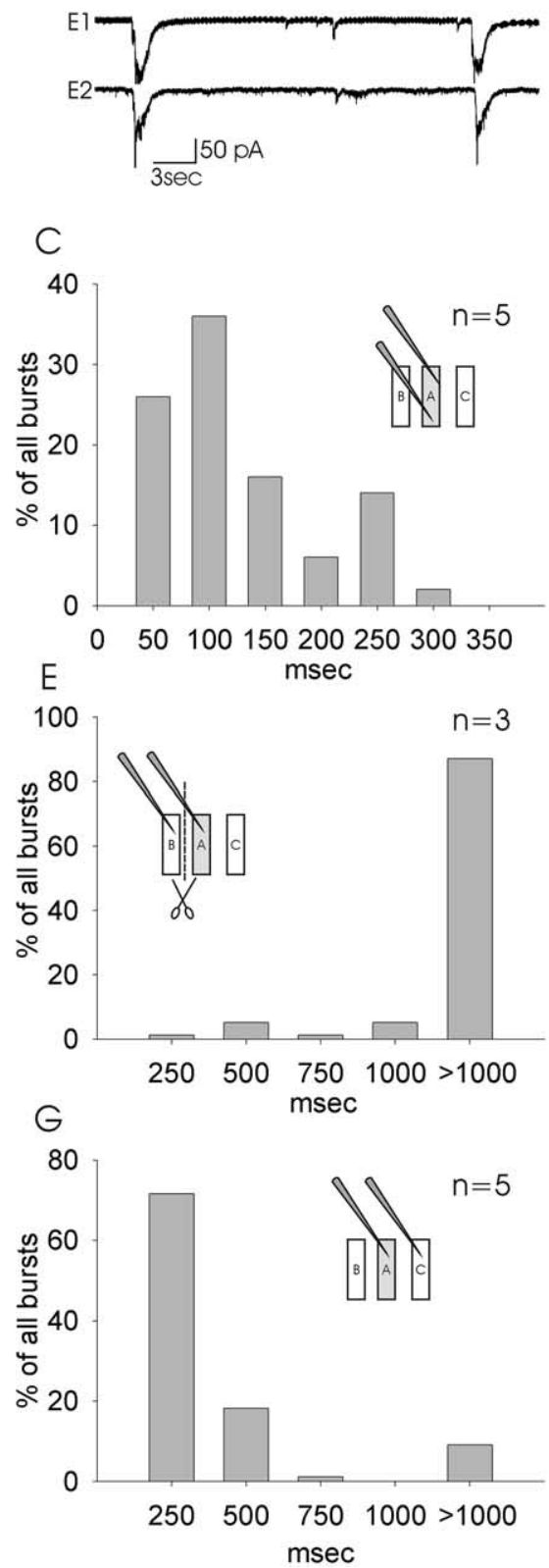

B
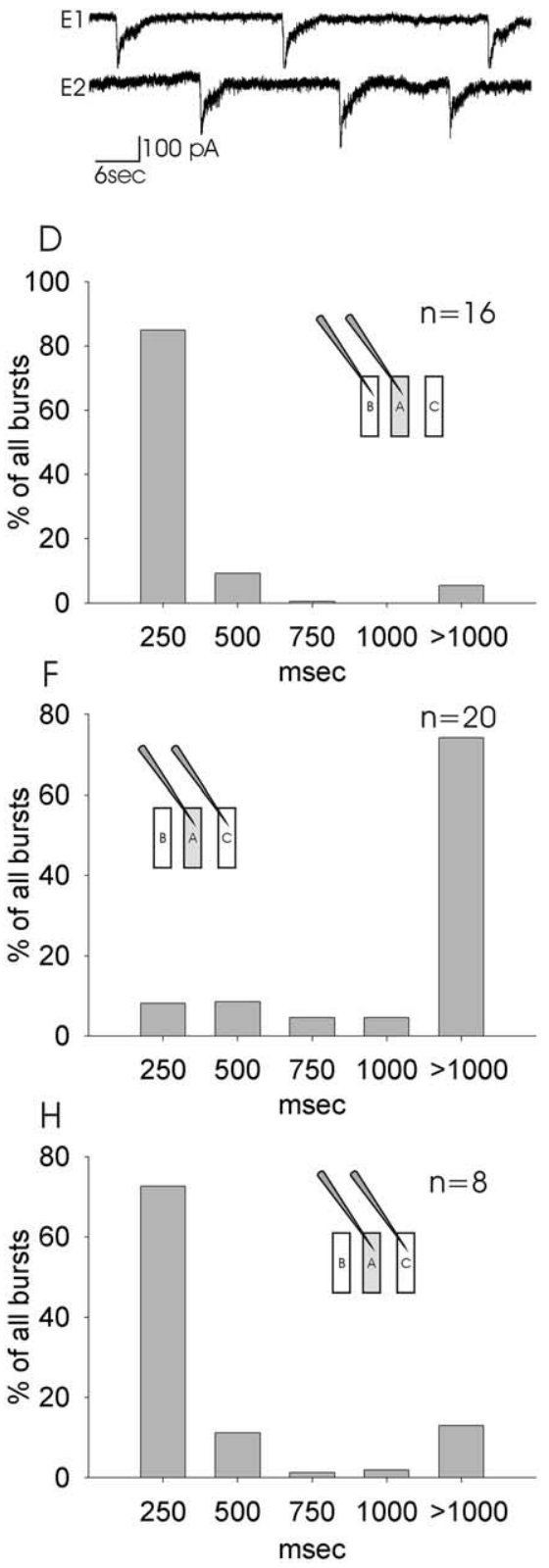

Figure 9. Evaluation of compartment connectivity with paired patch-clamp recordings. $\boldsymbol{A}, \boldsymbol{B}$, Connectivity within $13-$ to 16-d-old cultures was assessed by recording simultaneously two neurons. Synchronous network activity lead to barrages of synaptic currents that were either synchronized between both neurons $(\boldsymbol{A})$ or occurred out-of-phase and often with different frequency $(\boldsymbol{B})$. C, In simultaneously recorded neurons located at two distant sites of the same compartment (i.e., within 1 network), the onset of synchronous network activity occurred in both cells within a $250 \mathrm{~ms}$ interval. Based on these recordings, the bin width for the following histograms were set to $250 \mathrm{~ms}$ ( $n=5$ cultures, 1 pair per culture; see indications of culture number in the top right side of each histogram in this figure). $\boldsymbol{D}$, All A-B pairs (neurons in compartments separated by $1.5 \mathrm{~mm}$ ) showed synchronous burst activity (most bursts in the two recorded cells fell within a 250 ms interval, as shown in $\boldsymbol{A}$ ). $\boldsymbol{E}, \mathrm{A}-$ B pairs (neurons in compartments separated by $1.5 \mathrm{~mm}$ ) showed no synchronous burst activity (delay $>1 \mathrm{~s}$, as shown in $\boldsymbol{B}$ ) if the initially interconnected networks were mechanically separated. $\boldsymbol{F}, \boldsymbol{G}$, From the 25 recorded A-C neuronal pairs (neurons in compartments separated by $2.0 \mathrm{~mm}$ ), 20 were asynchronous (F; delay $>1 \mathrm{~s}$ ), and five pairs (G) presented synchronous burst activity. $\boldsymbol{H}$, After blockade of $\mathrm{AABA}_{\mathrm{A}}$ ergic and glutamatergic receptor activity until 13 DIV, all A-C pairs presented synchronous burst activity at 13-16 DIV.

cluding the stabilization of these developing synapses between late-coming axons and target neurons. An alternative possibility is that the activation of silent synapses prevents additional innervation by some unknown molecular mechanism. This seems less likely because axonal growth occurs beyond the initial period of synaptogenesis during experience-dependent refinement of synaptic connections (Katz and Shatz, 1996). However, it is possible 
that the activation of silent synapses in a leading growth cone prevents additional axonal elongation without inhibiting local axonal collateral growth.

During early brain development, the cortex is generated in a rostral-to-caudal and a lateral-to-medial gradient. Because of this, axons from different regions will reach their target areas at different time points. The present results suggest that silent synapses play a significant part in the context of the formation of corticocortical and probably also on thalamocortical connections. The initial formation of functional silent synaptic connections might allow cortical and thalamic axons to innervate a maximum of their appropriate target areas in the absence of any Hebbian-like competition. Then largescale synchronized activity facilitates the AMPAfication of preformed synapses globally throughout the entire structure at a defined point in time. In this way, all synaptic connections are activated in synchrony, guaranteeing equal starting conditions for all of them when activity-dependent refinement processes begin to operate.

One may argue that the discontinuity between cortical networks created in culture is not typical for the neonatal normal developing neocortex, in which waves of synchronous activity propagate continuously throughout the entire cortex and hippocampus (Garaschuk et al., 2000). However, it is feasible to imagine that regions with exacerbated asynchrony in network development may be accidentally created by localized trauma and other localized developmental disturbances. In this specific developmental time window that would generate disconnections, asynchrony that might not be repaired later.

In summary, we show here that a sequential formation of functional glutamatergic synapses might play a primordial role in determining the architecture of cortical and cortical-like networks. During the early network phase, connections grow to their targets and synchronized network depolarization activates immature synapses simultaneously. These two mechanisms (synchronous activity and silent synapses) act together to prevent a premature onset of regressive developmental changes as a consequence of heterosynaptic competition.

\section{References}

Bardoni R, Magherini PC, MacDermott AB (1998) NMDA EPSCs at glutamatergic synapses in the spinal cord dorsal horn of the postnatal rat. J Neurosci 18:6558-6567.

Ben-Ari Y (2001) Developing networks play a similar melody. Trends Neurosci 24:353-360.

Ben-Ari Y, Khazipov R, Leinekugel X, Caillard O, Gaiarsa JL (1997) GABA NMDA and AMPA receptors: a developmentally regulated "menage a trois." Trends Neurosci 20:523-529.

Bottenstein JE, Sato GH (1979) Growth of a rat neuroblastoma cell line in serum-free supplemented medium. Proc Natl Acad Sci USA 76:514-517.

Carroll RC, Lissin DV, von Zastrow M, Nicoll RA, Malenka RC (1999) Rapid redistribution of glutamate receptors contributes to long-term depression in hippocampal cultures. Nat Neurosci 2:454-460.

Craig AM, Blackstone CD, Huganir RL, Banker G (1993) The distribution of glutamate receptors in cultured rat hippocampal neurons: postsynaptic clustering of AMPA-selective subunits. Neuron 10:1055-1068.

de Lima AD, Voigt T (1999) Astroglia inhibit the proliferation of neocortical cells and prevent the generation of small GABAergic neurons in vitro. Eur J Neurosci 11:3845-3856.

Durand GM, Kovalchuk Y, Konnerth A (1996) Long-term potentiation and functional synapse induction in developing hippocampus. Nature 381:71-75.

Feldman DE, Nicoll RA, Malenka RC (1999) Synaptic plasticity at thalamocortical synapses in developing rat somatosensory cortex: LTP, LTD, and silent synapses. J Neurobiol 41:92-101.

Feller MB (1999) Spontaneous correlated activity in developing neural circuits. Neuron 22:653-656.

Garaschuk O, Hanse E, Konnerth A (1998) Developmental profile and synaptic origin of early network oscillations in the CA1 region of rat neonatal hippocampus. J Physiol (Lond) 507:219-236.
Garaschuk O, Linn J, Eilers J, Konnerth A (2000) Large-scale oscillatory calcium waves in the immature cortex. Nat Neurosci 3:452-459.

Gomperts SN, Rao A, Craig AM, Malenka RC, Nicoll RA (1998) Postsynaptically silent synapses in single neuron cultures. Neuron 21:1443-1451.

Hall RA, Soderling TR (1997) Differential surface expression and phosphorylation of the $N$-methyl-D-aspartate receptor subunits NR1 and NR2 in cultured hippocampal neurons. J Biol Chem 272:4135-4140.

Hsia AY, Malenka RC, Nicoll RA (1998) Development of excitatory circuitry in the hippocampus. J Neurophysiol 79:2013-2024.

Isaac JT (2003) Postsynaptic silent synapses: evidence and mechanisms. Neuropharmacology 45:450-460.

Isaac JT, Nicoll RA, Malenka RC (1995) Evidence for silent synapses: implications for the expression of LTP. Neuron 15:427-434.

Isaac JT, Crair MC, Nicoll RA, Malenka RC (1997) Silent synapses during development of thalamocortical inputs. Neuron 18:269-280.

Katz LC, Shatz CJ (1996) Synaptic activity and the construction of cortical circuits. Science 274:1133-1138.

Li P, Zhuo M (1998) Silent glutamatergic synapses and nociception in mammalian spinal cord. Nature 393:695-698.

Liao D, Hessler NA, Malinow R (1995) Activation of postsynaptically silent synapses during pairing-induced LTP in CA1 region of hippocampal slice. Nature 375:400-404.

Liao D, Zhang X, O’Brien R, Ehlers MD, Huganir RL (1999) Regulation of morphological postsynaptic silent synapses in developing hippocampal neurons. Nat Neurosci 2:37-43.

Liao D, Scannevin RH, Huganir R (2001) Activation of silent synapses by rapid activity-dependent synaptic recruitment of AMPA receptors. J Neurosci 21:6008-6017.

Losi G, Prybylowski K, Fu Z, Luo JH, Vicini S (2002) Silent synapses in developing cerebellar granule neurons. J Neurophysiol 87:1263-1270.

Lu W, Man H, Ju W, Trimble WS, MacDonald JF, Wang YT (2001) Activation of synaptic NMDA receptors induces membrane insertion of new AMPA receptors and LTP in cultured hippocampal neurons. Neuron 29:243-254.

Malenka RC, Nicoll RA (1997) Silent synapses speak up. Neuron 19:473-476.

Murphy TH, Blatter LA, Wier WG, Baraban JM (1992) Spontaneous synchronous synaptic calcium transients in cultured cortical neurons. J Neurosci 12:4834-4845.

O’Donovan MJ (1999) The origin of spontaneous activity in developing networks of the vertebrate nervous system. Curr Opin Neurobiol 9:94-104.

Opitz T, de Lima AD, Voigt T (2002) Spontaneous development of synchronous oscillatory activity during maturation of cortical networks in vitro. J Neurophysiol 88:2196-2206.

Petralia RS, Esteban JA, Wang YX, Partridge JG, Zhao HM, Wenthold RJ, Malinow R (1999) Selective acquisition of AMPA receptors over postnatal development suggests a molecular basis for silent synapses. Nat Neurosci 2:31-36.

Pickard L, Noel J, Henley JM, Collingridge GL, Molnar E (2000) Developmental changes in synaptic AMPA and NMDA receptor distribution and AMPA receptor subunit composition in living hippocampal neurons. J Neurosci 20:7922-7931.

Rumpel S, Hatt H, Gottmann K (1998) Silent synapses in the developing rat visual cortex: evidence for postsynaptic expression of synaptic plasticity. J Neurosci 18:8863-8874.

Schwindt PC, Spain WJ, Foehring RC, Chubb MC, Crill WE (1988) Slow conductances in neurons from cat sensorimotor cortex in vitro and their role in slow excitability changes. J Neurophysiol 59:450-467.

Shi SH, Hayashi Y, Petralia RS, Zaman SH, Wenthold RJ, Svoboda K, Malinow R (1999) Rapid spine delivery and redistribution of AMPA receptors after synaptic NMDA receptor activation. Science 284:1811-1816.

Voigt T, Baier H, de Lima AD (1997) Synchronization of calcium activity promotes survival of individual rat neocortical neurons in early development. Eur J Neurosci 9:990-999.

Voigt T, Opitz T, de Lima AD (2001) Synchronous oscillatory activity in immature cortical network is driven by GABAergic preplate neurons. J Neurosci 21:8895-8905.

Voronin LL, Cherubini E (2003) "Presynaptic silence" may be golden. Neuropharmacology 45:439-449.

Wong ROL (1998) Calcium imaging and multielectrode recordings of global patterns of activity in the developing nervous system. Histochem J 30:217-229.

Wu GY, Malinow R, Cline HT (1996) Maturation of a central glutamatergic synapse. Science 274:972-976. 